\title{
Thiocyanate-Treated Perovskite-Nanocrystal-Based Light-Emitting Diodes with Insight in Efficiency Roll-Off
}

\author{
Fang Chen ${ }^{1,2}$, Karunakara Moorthy Boopathi ${ }^{3}$, Muhammad Imran $^{3}$, Simone Lauciello $^{3}$ and \\ Marco Salerno $4, *$ (D) \\ 1 Optoelectronics, Istituto Italiano di Tecnologia, via Morego 30, 16163 Genova, Italy; fang.chen@iit.it \\ 2 Dipartimento di Chimica e Chimica Industriale, Università degli Studi di Genova, via Dodecaneso 31, \\ 16146 Genova, Italy \\ 3 Nanochemistry Department, Istituto Italiano di Tecnologia, via Morego 30, 16163 Genova, Italy; \\ karunakara.boopathi@iit.it (K.M.B.); muhammad.imran@iit.it (M.I.); simone.lauciello@iit.it (S.L.) \\ 4 Materials Characterization Facility, Istituto Italiano di Tecnologia, via Morego 30, 16163 Genova, Italy \\ * Correspondence: marco.salerno@iit.it; Tel.: +39-010-2896-885
}

Received: 13 December 2019; Accepted: 10 January 2020; Published: 13 January 2020

\begin{abstract}
Light emitting diodes (LED) based on halide perovskite nanocrystals (NC) have received widespread attention in recent years. In pArticular, LEDs based on $\mathrm{CsPbBr}_{3} \mathrm{NCs}$ were the object of special interest. Here, we report for the first time green LED based on $\mathrm{CsPbBr}_{3} \mathrm{NCs}$ treated with ammonium thiocyanate solution before purification with polar solvent. The champion device fabricated based on the treated $\mathrm{CsPbBr}_{3} \mathrm{NCs}$ showed high efficiency and high stability during operation as well as during storage. A study on morphology and current distribution of NC films under applied voltages was carried out by conductive atomic force microscopy, giving a hint on efficiency roll-off. The current work provides a facile way to treat sensitive perovskite NCs and to fabricate perovskite NC-based LED with high stability. Moreover, the results shed new light on the relation between film morphology and device performance and on the possible mechanism of efficiency roll-off in NC LED.
\end{abstract}

Keywords: perovskite nanocrystal; light emitting diodes; thiocyanate; efficiency roll-off; conductive atomic force microscopy

\section{Introduction}

All-inorganic halide perovskite has attracted much attention since successful synthesis of $\mathrm{CsPbX}_{3}$ $(\mathrm{X}=\mathrm{Cl}, \mathrm{Br}, \mathrm{I}) \mathrm{NC}$ in 2015 [1]. Owing to their excellent photoluminescence (PL) performance [2] and facile synthesis in solution [3], $\mathrm{CsPbX}_{3} \mathrm{NCs}$, especially $\mathrm{CsPbBr}_{3}$, are currently the subject of wide exploration in LED [4]. In comparison with their contemporary organic-inorganic hybrid halide perovskites, $\mathrm{CsPbX}_{3} \mathrm{NCs}$ exhibit higher stability and narrower emission width [5]. Currently, the best halide perovskite nanocrystal-based LED with green emission has achieved a maximum external quantum efficiency (EQE) of $16.48 \%$, based on NCs containing mixed cations of cesium and formamidine [6]. However, progress made in all-inorganic halide-perovskite nanocrystal-based LED relatively lags behind.

Various synthesis strategies for $\mathrm{CsPbBr} 3$ NCs have been extensively exploited based on different source materials, ligands and reaction conditions. Continuous progresses are being made in applying $\mathrm{CsPbBr}_{3} \mathrm{NCs}$ synthesized by different methods into LED fabrication. Firstly, based on the first synthesis route of $\mathrm{CsPbBr}_{3} \mathrm{NCs}$ [1], $\mathrm{CsPbBr} 3 \mathrm{NCs}$ synthesized by $\mathrm{PbBr}_{2}$ and $\mathrm{Cs}_{2} \mathrm{CO}_{3}$ have obtained a fast development in EQE of corresponding LEDs. For example, EQEs of 0.26\% [7] and 5.7\% [8] 
have been achieved by optimizing transporting layers based on the initial EQE of $0.026 \%$ [7]. By optimizing ligand washing process, the EQE was increased to 1.7\% [9]. Via ligand exchange in post-synthesis, EQE of 3.0\% [10] and 4.33\% [11] were realized, respectively, with didodecyldimethyl ammonium bromide (DDAB) and conjugation molecular. By combination of ligand exchange with DDAB and optimized ligand washing strategy, the EQE was further increased up to more than $8.0 \%$ [12]. However, it is tedious to perform ligand exchange and the productivity of NCs after ligand exchange is rather low. Application of the $\mathrm{CsPbBr}_{3} \mathrm{NCs}$ into LEDs with inverted structure has developed from initial EQE of $0.19 \%$ [13] up to $4.63 \%$ [14] by optimizing band alignment of transporting layers and introducing localized surface plasma. Secondly, when the source material of cesium is replaced with cesium stearate, performance of corresponding LEDs has also received a steady enhancement. For instance, starting from initial EQE of $0.12 \%$ [15], further works have increased the EQE to $2.21 \%$ by forming composite with $\mathrm{CsPb}_{2} \mathrm{Br}_{5}$ [16], and $6.27 \%$ by rational washing procedure of the $\mathrm{CsPbBr}_{3}$ NCs [17]. Trial of the $\mathrm{CsPbBr}_{3} \mathrm{NCs}$ with DDAB ligand exchange in inverted LED structure has obtained a maximum EQE of $0.58 \%$ [18]. In addition, cesium acetate was also applied into synthesis of $\mathrm{CsPbBr}_{3} \mathrm{NCs}$. Together with octylphosphonic acid as ligand, EQE of 6.5\% [19] was achieved. Thirdly, some work involved the use of new types of halide sources in synthesis of the $\mathrm{CsPbBr}_{3} \mathrm{NCs}$, e.g., tetraoctylammonium bromide (TOABr), $\mathrm{NH}_{4} \mathrm{Br}$, trioctylphosphine- $\mathrm{Br}_{2}$ and benzoyl bromide. For instance, $\mathrm{LED}$ based on $\mathrm{CsPbBr}_{3} \mathrm{NC}$ s prepared with $\mathrm{TOABr}$ as halide source and oleic acid as ligand obtained a maximum EQE of $0.325 \%$ [20]. Through combining mixed cation, ligand exchange, rational washing and surface pAssivation in treatment of $\mathrm{CsPbBr}_{3} \mathrm{NCs}$, LED based on the $\mathrm{CsPbBr}_{3} \mathrm{NCs}$, synthesized with $\mathrm{PbBr}_{2}, \mathrm{Cs}_{2} \mathrm{CO}_{3}$ and $\mathrm{TOABr}$, has obtained a maximum EQE of $16.48 \%$ [6]. In another case, LEDs based on CBP NCs using NH4Br [21] and trioctylphosphine- $\mathrm{Br}_{2}$ [22] in synthesis have also been reported, with maximum EQEs at $1.2 \%$ and $2.5 \%$, respectively. However, despite that CBP NCs prepared with benzoyl bromide as halide source have achieved a high PL quantum yield (PLQY) of $92 \%$ in solution [23], there has been no report on corresponding LED.

Passivation of defects in either perovskite thin film or perovskite NCs play an important role. For example, with methylammonium bromide additive pAssivating non-radiative defect sites in $\mathrm{CsPbBr}_{3}$ thin film, a milestone EQE of $20.3 \%$ was realized [24]. For another example, with inorganic halide ionic compounds pAssivating $\mathrm{CsPbBr}_{3} \mathrm{NCs}$ before purification with anti-solvent, the up-to-date highest EQE for perovskite NCs has been achieved [6]. Salts containing thiocyanate (SCN) have been demonstrated to be able to increase grain size and reduce defects in perovskite thin film [25] and have shown beneficial effects on stability [26], device performance and suppression of hysteresis in solar cell [27]. SCN, as a kind of pseudohalide, has also presented efficient pAssivation effect on perovskite NCs [28]. For instance, Alivisatos and coworkers reported that SCN improves PLQY and stability of CBP NCs by surface pAssivation [29], and proposed a principle to pAssivate $\mathrm{CsPbBr}_{3} \mathrm{NCs}$ with softer, anionic and X-type Lewis bases [30]. Recently, Lu et al. [31] reported red-emitting LEDs based on

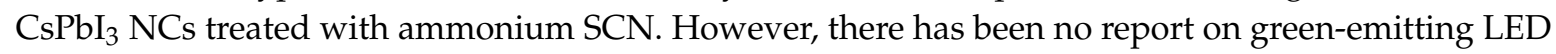
based on SCN-treated $\mathrm{CsPbr}_{3} \mathrm{NCs}$.

Efficiency roll-off at high current density in perovskite LED has started to receive attention. Auger recombination has been determined as a reason for this effect [32], and some solutions have been proposed correspondingly. For instance, core-shell structure was shown to be beneficial to increase threshold current of efficiency roll-off in QD LED [33]. In another work, in 2D perovskite with funnel structure, the roll-off was demonstrated to be reduced through increasing content ratio of low-bandgap perovskite [32], which concentrates the injected electrons/holes and plays the major role in light emission. For 3D perovskite NCs, material degradation [34] and destruction of film morphology [35] at high current density were predicted to play critical roles in the roll-off. However, there has been no direct proof reported for this hypothesis.

In this work, green LEDs based on $\mathrm{CsPbBr}_{3}$ treated with ammonium SCN solution before purification with polar solvent were fabricated for the first time. The LEDs show high efficiency compared with LEDs based on $\mathrm{CsPbBr}_{3} \mathrm{NCs}$ without DDAB ligand exchange, which is tedious and 
of low productivity in NC preparation [36]. The champion device also shows high stability during operation and during storage. Investigations on morphology and current distribution of NC films with structure similar to the final device, under applied voltages, were performed by conductive atomic force microscopy (AFM), to illustrate the possible mechanisms of better performance in the champion device. With this work, we provide a facile treatment for achieving stable perovskite NC LED and present some insight in the possible mechanism of efficiency roll-off in NC LED.

\section{Materials and Methods}

\subsection{Materials}

Ethyl acetate, ammonium thiocyanate and 1-Butanol were purchased from Sigma-Aldrich (Milan, Italy) and were stored in glovebox. NC solutions synthesized according to the previous report [23] were treated in such a way that $300 \mu \mathrm{L}$ as-prepared $\mathrm{CsPbBr}_{3} \mathrm{NC}$ solution was firstly added into a vial. Secondly, an amount of ammonium thiocyanate (SCN) solution, for example, 10, 30 or $50 \mu \mathrm{L}$, was dropped into the vial. Thirdly, $900 \mu \mathrm{L}$ EA was gradually added into the solution, and the obtained solution was left under hood for $5 \mathrm{~min}$. Then, the treated $\mathrm{CsPbBr}_{3} \mathrm{NC}$ solution was centrifuged at $6000 \mathrm{rpm}$ for $10 \mathrm{~min}$. The supernatant was discarded, and the precipitate was re-dispersed in $300 \mu \mathrm{L}$ hexane for film and device fabrication. Here, SCN solution was prepared by dissolving $10 \mathrm{mg}$ ammonium thiocyanate in a mixture of $4 \mathrm{~mL}$ 1-butanol and $6 \mathrm{~mL}$ toluene.

\subsection{Device Preparation}

LEDs were fabricated on pAtterned substrates of glass coated with $\sim 200 \mathrm{~nm}$ thick indium tin oxide (ITO). The substrates were cleaned in an ultrasonic bath using detergent, deionized water, isopropanol and deionized water, sequentially. Prior to depositing hole transporting layers, the ITO glass substrates were further treated with oxygen plasma for $300 \mathrm{~s}$ at $30 \mathrm{~W}$. A layer of poly(ethylene dioxythiophene): polystyrenesulphonate (PEDOT:PSS) was spin-coated onto the cleaned ITO glass substrates at $4000 \mathrm{rpm}$ and annealed at $150{ }^{\circ} \mathrm{C}$ for $30 \mathrm{~min}$ in hood. Poly[ $N, N^{\prime}$-bis(4-butylphenyl)- $N, N^{\prime}$-bisphenylbenzidine $]$ (poly-TPD) was spin-coated onto the PEDOT:PSS layer at $5000 \mathrm{rpm}$ and annealed at $120^{\circ} \mathrm{C}$ for $20 \mathrm{~min}$ in glovebox (GB). The total thickness of PEDOT:PSS and poly-TPD was $\sim 60 \mathrm{~nm}$. Here, the poly-TPD solution was prepared in chlorobenzene at $8 \mathrm{~g} / \mathrm{L}$ in $\mathrm{GB}$. As the film cool down, $\mathrm{CsPbBr}_{3} \mathrm{NC}$ solutions were spin-casted at $2000 \mathrm{rpm}$ and annealed at $70{ }^{\circ} \mathrm{C}$ for $15 \mathrm{~min}$. The thickness was $\sim 30 \mathrm{~nm}$. Then, electron transporting layer 1,3,5-Tris(1-phenyl-1H-benzimidazol-2-yl)benzene (TPBi) $(\sim 45 \mathrm{~nm})$ and electrode $\mathrm{LiF}(\sim 1.5 \mathrm{~nm})$ and $\mathrm{Al}(\sim 100 \mathrm{~nm})$ were thermal evaporated at rate of $1.5,0.2,2 \mathrm{~nm} / \mathrm{s}$, respectively. Finally, the devices were encapsulated with cover glasses and encapsulation oil. The devices were treated by UV light for 15 min to solidify the encapsulation oil in GB.

\subsection{Characterization}

The NC solutions were characterized by a transmission electron microscope (TEM) JEOL-1100 (Jeol, Tokyo, Japan) at $100 \mathrm{kV}$ and low resolution (after drop-casting onto carbon-coated copper grids) and by X-ray diffraction (XRD) on a Empyrean X-ray diffractometer (PANanalytical, Almelo, the Netherlands) equipped with a $1.8 \mathrm{~kW} \mathrm{Cu} \mathrm{K} \alpha$ ceramic X-ray tube and a PIXcel ${ }^{3 \mathrm{D}} 2 \times 2$ area detector, operating at $45 \mathrm{kV}$ and $40 \mathrm{~mA}$ (after drop-casting onto silicon wafer). The XRD pAtterns were collected under ambient conditions using pArallel beam geometry and symmetric reflection mode, and data analysis was conducted using the HighScore 4.1 software from PANanalytical.

Films with similar structure to device were characterized by Cary 5000 UV-vis-NIR spectrophotometer (Varian, pAlo Alto, CA, USA), fluorescence spectrometer FLS920 (Edinburgh Instruments, Kirkton Campus, UK) at an excitation wavelength (Xenon lamp and monochromator) of $350 \mathrm{~nm}$; A1 confocal fluorescence microscopy (Nikon, Shinjuku, Japan) with a laser excitation wavelength of $401 \mathrm{~nm}$; and MFP3D AFM system with ORCA mode, with probes RMN-12PT400B (Bruker, Billerica, MA, US) coated with platinum. Different voltages of $-3 \mathrm{~V},-5 \mathrm{~V}$ and $-7 \mathrm{~V}$ were 
applied on the tip. The sample used for C-AFM consisted of ITO/PEDOT:PSS/Poly-TPD/perovskite NCs, which follows similar structure to the device. In order to simulate the work mode of a full device, negative voltage was applied on the tip to inject electrons into the NCs while the ITO layer is electrically connected to the circuit to provide holes.

The cross-section of the device was measured by field-emission scanning electron microscope (SEM) JSM7500LA (Jeol, Tokyo, Japan) operating at $20 \mathrm{kV}$.

The current-voltage-luminance measurement was performed using a Keithley 2410 source-measure unit and an Agilent 34410A multi-meter coupled to a calibrated PDA 100A Si switchable gain detector from Thorlabs. The output of the Si detector was converted into power (photon flux) using a $50 \Omega$ load resistance and the responsivity of the detector. The external quantum efficiency (EQE) was calculated as the ratio of the photon flux and the driving current of the device. The EL spectra of the devices were collected by an Ocean Optics HR4000+ spectrometer.

\section{Results and Discussion}

$\mathrm{CsPbBr}_{3} \mathrm{NCs}$ were prepared with lead acetate trihydrate, cesium carbonate and benzoyl bromine as sources of lead, cesium and bromide, respectively. The synthesized $\mathrm{CsPbBr}_{3} \mathrm{NC}$ solution was treated with different amount of SCN solution before purification with EA. According to the added amount of SCN solution including $0,10,30$ and $50 \mu \mathrm{L}$, the $\mathrm{CsPbBr}_{3} \mathrm{NC}$ solutions were labeled as $0 \mathrm{SCN}, 10 \mathrm{SCN}$, $30 \mathrm{SCN}$ and $50 \mathrm{SCN}$, respectively. The detailed treatment method can be found in the experimental section. Additionally, in the Supporting Information file, some routine characterization of the NCs is also included, such as TEM images (See Supplementary Figure S1a-j), showing an average size in the 10-12 nm range, and XRD pAtterns (Figure S1k), showing that all the XRD peaks are similar and can be assigned to $\mathrm{CsPbBr}$. The sulfur element contained in SCN was not detectable [37] by XPS and by HAADF-EDS in 30SCN. This may be due to either the sulfur contents being below detection threshold or to its probable overlap with the signal of lead.

With the treated $\mathrm{CsPbBr}_{3} \mathrm{NCs}$ used as emissive material, we fabricated and characterized corresponding LEDs. Figure 1a presents a schematic diagram of the LED structure, ITO/PEDOT:PSS/poly-TPD/CsPbBr 3 NC/TPBi/LiF/Al. The SEM cross-section of 30SCN device is shown in Figure S2. Figure 1b displays variation of current density with increasing voltage for the three devices. 50SCN presents higher leakage than $10 \mathrm{SCN}$ and $30 \mathrm{SCN}$, suggesting higher conductivity of 50SCN NC film due to less organic than $10 \mathrm{SCN}$ and 30SCN NC films. At voltage higher than $7 \mathrm{~V}$, $30 \mathrm{SCN}$ shows higher current density than $10 \mathrm{SCN}$ and $50 \mathrm{SCN}$. In the current density plot of 30SCN, there is a turning point around $4.3 \mathrm{~V}$, indicating variation of growth rate of the current density with increasing voltage (Figure S3a).

Figure 1c displays variation of luminance with increasing current density. It is clear that $30 \mathrm{SCN}$ shows higher luminance than $10 \mathrm{SCN}$ and $50 \mathrm{SCN}$ across the whole range of the current density and reaches the highest level of $2986 \mathrm{~cd} / \mathrm{m}^{2}$ at $187 \mathrm{~mA} / \mathrm{cm}^{2}$. 10SCN reaches the highest luminance of 1018 $\mathrm{cd} / \mathrm{m}^{2}$ at $461 \mathrm{~mA} / \mathrm{cm}^{2}$, and $50 \mathrm{SCN}$ reaches the highest luminance of $890 \mathrm{~cd} / \mathrm{m}^{2}$ at $80 \mathrm{~mA} / \mathrm{cm}^{2} .10 \mathrm{SCN}$ has the highest turn-on voltage at $4.4 \mathrm{~V}$ with luminance at $1 \mathrm{~cd} / \mathrm{m}^{2}$, while turn-on voltages of $30 \mathrm{SCN}$ and 50SCN are at $3.2 \mathrm{~V}$ and $3.3 \mathrm{~V}$, indicating less insulating organic ligands in the $\mathrm{CsPbBr}_{3} \mathrm{NC}$ film with increasing SCN additive during treatment. Although $30 \mathrm{SCN}$ shows a relatively slower decrease of luminance when the current density is less than $300 \mathrm{~mA} / \mathrm{cm}^{2}$, all the three devices present luminance roll-off after reaching the respective highest luminance.

Figure $1 \mathrm{~d}$ displays variation of EQE with increasing current density. $30 \mathrm{SCN}$ presents the highest EQE of $1.2 \%$ at $32 \mathrm{~mA} / \mathrm{cm}^{2}$ or $5.5 \mathrm{~V}$ and the highest current efficiency of $4.42 \mathrm{~cd} / \mathrm{A}$ among the three devices. The luminance at the maximum EQE is $1404 \mathrm{~cd} / \mathrm{m}^{2}$, which is relatively high among the LEDs based on $\mathrm{CsPbBr}_{3} \mathrm{NCs}$ with organic transporting layers (Table S1). In contrast, 10SCN and 50SCN show maximal EQEs of $0.06 \%$ at $380 \mathrm{~mA} / \mathrm{cm}^{2}$ and $0.68 \%$ at $12 \mathrm{~mA} / \mathrm{cm}^{2}$, respectively. Despite that, the trend of the EQE and current density is similar to the relation of luminance intensity and current density in Figure 1c; efficiency roll-off is more outstanding, especially in 30SCN and 50SCN. Given that 
current densities corresponding to the highest luminance or EQE of each device are different, it can be inferred that Auger recombination is not necessarily the reason for the efficiency roll-off in current cases. The device performance of OSCN is displayed in Figure S4.
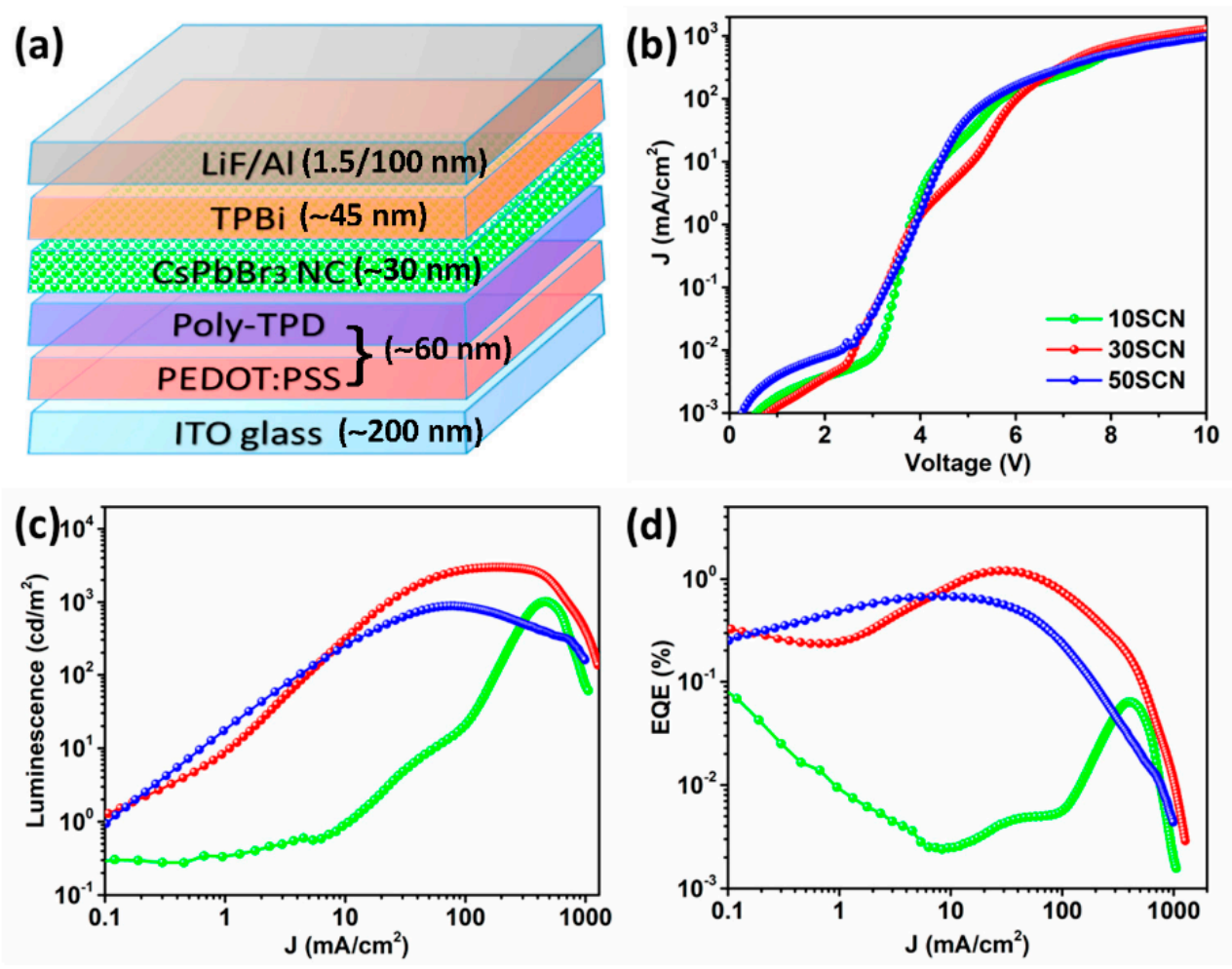

Figure 1. Characterization of LED performance. (a) Diagram of the LED structure; (b) current density as a function of driving voltage; (c) luminescence and (d) external quantum efficiency (EQE) as a function of current density in the NC LEDs.

In order to explain the better device performance of the $30 \mathrm{SCN}$, the NC solutions were spin-coated onto substrates coated with ITO/PEDOT:PSS/poly-TPD. The film samples show similar optical characterization results on the multi-layer substrates (see Figure S5).

The film samples were then characterized by confocal microscopy. Figure 2 presents PL mapping images and PL spectra of selected areas on the images. The PL mapping image of 30SCN (Figure 2c) looks smoother and more homogeneous, in accord with the quantitative analysis result (Figure 2g) that the PL intensities of different selected areas with a diameter of $35 \mu \mathrm{m}$ in 30SCN film sample show smaller difference or more homogeneous NC distribution than those of the other three film samples. In contrast, there are a few rather bright pArts sparsely distributing in 10SCN and 50SCN film samples. The inhomogeneously bright areas may be caused by aggregation of NCs. Corresponding PL spectra of the selected areas in 10SCN and 50SCN film samples show relatively wide distribution in PL intensities, in accordance with the range of brightness showing in the PL mapping images. The spread in brightness can be regarded as striation defects and was demonstrated to be due to difference of surface tension and vapor pressure of organic residues and NCs coexisting in the film [19]. In other words, a proper ratio between ligands and $\mathrm{CsPbBr}_{3} \mathrm{NCs}$ is beneficial for forming smooth $\mathrm{NC}$ film. This also indicates that the proportion of ligands and $\mathrm{CsPbBr}_{3} \mathrm{NCs}$ in $30 \mathrm{SCN}$ is at an optimal level. In the PL mapping image of OSCN (Figure 2a), one can clearly see bright dots distributing across the film, making the film look rough. This may be due to aggregation of the $\mathrm{CsPbBr}_{3} \mathrm{NCs}$ or growth of some $\mathrm{CsPbBr}_{3} \mathrm{NCs}$, as demonstrated in another work in preparation showing that the untreated $\mathrm{CsPbBr}_{3} \mathrm{NCs}$ in film are prone to grow into larger size due to desorption of ligands. 

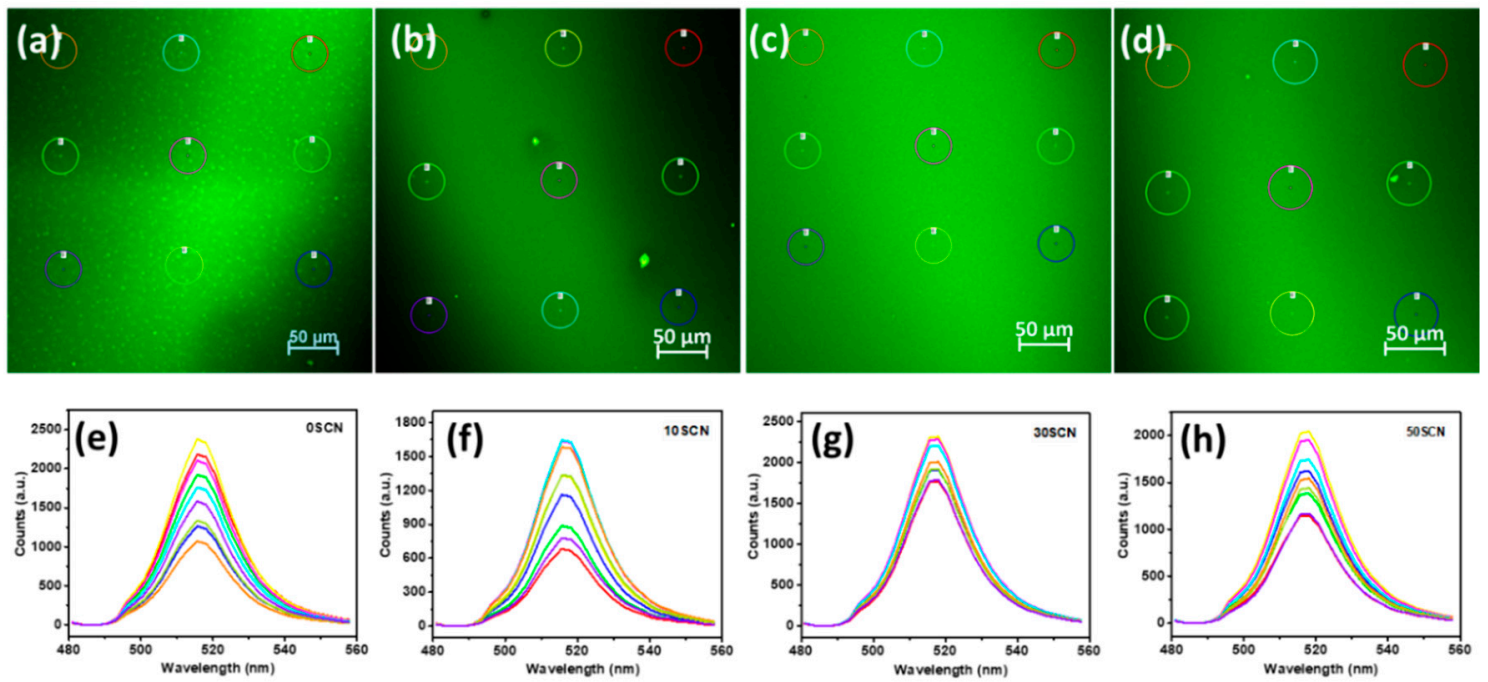

Figure 2. PL mapping images of NC films based on (a) 0SCN, (b) 10SCN, (c) 30SCN and (d) 50SCN NC solutions; (e-h) PL spectra of selected areas in each of the corresponding PL mapping images.

The above results on films match well with the LED performance. For example, too many ligands in OSCN may explain the low current density and ineffective transport of injected carriers in the newly prepared LED, while there is strong leakage and emission from transporting layers, when large pArticles form after aging the device for some days (Figure S4). On the other hand, 10SCN shows the most inhomogeneous NC distribution on film, and the corresponding device displays the lowest efficiency. The 30SCN sample presents the best film homogeneity and the corresponding LED shows the highest luminance and EQE. Thus, the better device performance of the 30SCN may be attributed to both pAssivation effect of SCN, and the smooth and homogeneous NC film. Actually, both 30SCN and $50 S C N$ gave good quality films, but $50 \mathrm{SCN}$ film was worse than $30 \mathrm{SCN}$ one. This may indicate that proper amount of SCN may have pAssivation effect and be beneficial, and too much SCN is not good. Thus, for $10 \mathrm{SCN}$ and $30 \mathrm{SCN}$, the difference in LED performance can be attributed to difference of film morphology or quality.

Given that the above characterizations are performed on static films, we further tried to measure evolution of the films under voltage by conductive atomic force microscopy (C-AFM) [38]. Figure 3 shows topographical images and current mapping images of $10 \mathrm{SCN}$ and $30 \mathrm{SCN}$ under different applied voltages. In the case of $10 \mathrm{SCN}$, with increasing applied voltages during scanning, the selected scanning area becomes rougher and aggregation of the $\mathrm{CsPbBr}_{3} \mathrm{NCs}$ become increasingly serious. In specific, when the applied voltage increases from $3 \mathrm{~V}$ to $5 \mathrm{~V}$, more areas without coverage of NCs come into being with the root mean square roughness pArameter rising up from 2.9 to $3.7 \mathrm{~nm}$ (Figure $3 \mathrm{a}, \mathrm{b}$ ). When the voltage further increases to $7 \mathrm{~V}$, the uncovered regions become larger in area in spite of the lesser number of domains (Figure 3c). The measured roughness apparently stays on the same level $(\sim 3.6 \mathrm{~nm})$. Correspondingly, the current distribution follows the morphology evolution. At $3 \mathrm{~V}$, the whole film is at a low current level and the average current is $\sim 18 \mathrm{pA}$ (Figure $3 \mathrm{~d}$ ). At $5 \mathrm{~V}$, the overall current of the film increases according to the larger medium current (green yellow) and higher current (red) areas instead of lower current (blue white) areas (Figure 3e). The red areas of the current mapping image indicate higher current leakage than the other pArts and may represent absence of $\mathrm{CsPbBr}_{3} \mathrm{NCs}$, in line with the morphology image. When $7 \mathrm{~V}$ is applied during scanning, the distribution of red and blue-white domains change (Figure 3f). The red domains decrease in number and become larger in size, while the rest of the scanning area mainly lies on the blue-white current levels. This suggests stronger leakage through the uncovered areas and less current flow through the NC areas, supplying evidence for low efficient radiative recombination of injected carriers. This may explain the low luminance and EQE of $10 \mathrm{SCN}$. 


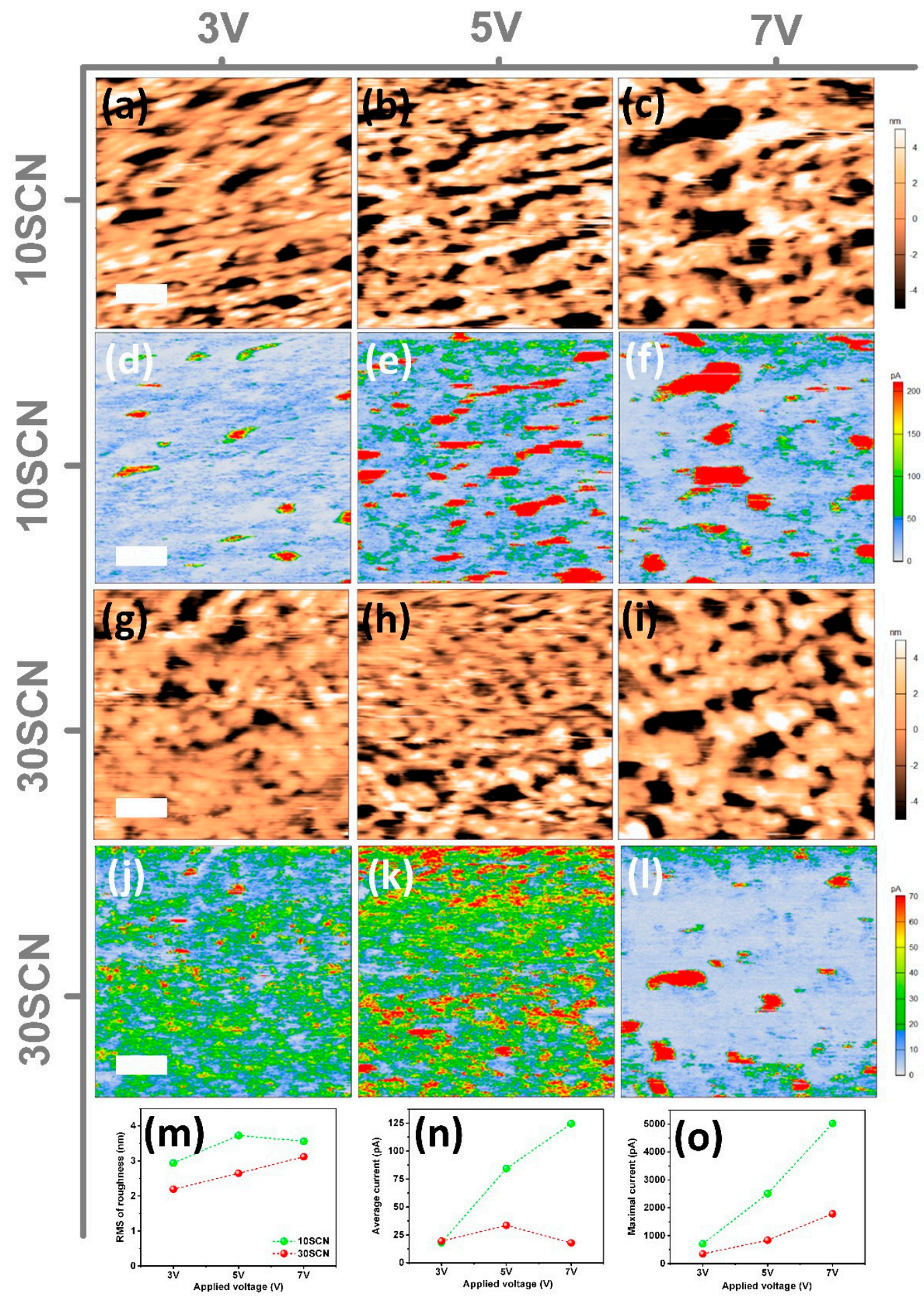

Figure 3. (a-c) Topography and (d-f) current mapping images of $10 \mathrm{SCN} N \mathrm{NC}$ film under $3 \mathrm{~V}, 5 \mathrm{~V}$ and $7 \mathrm{~V}$; (g-i) topography and (j-1) current mapping images of 30SCN NC film under $3 \mathrm{~V}, 5 \mathrm{~V}$ and $7 \mathrm{~V}$; comparison of $(\mathbf{m})$ roughness, $(\mathbf{n})$ average current, and (o) maximum current of $10 \mathrm{SCN}$ and $30 \mathrm{SCN}$ NC films under voltages. The scale bars are $200 \mathrm{~nm}$.

In the case of 30SCN, the overall roughness of the film is less than that of the $10 \mathrm{SCN}$ at different voltages (Figure 3g-i). With applied voltages increasing from $3 \mathrm{~V}$ to $7 \mathrm{~V}$, the roughness of the $30 \mathrm{SCN}$ film increases monotonously from $2.2 \mathrm{~nm}$ to $3.1 \mathrm{~nm}$ (Figure $3 \mathrm{~m}$ ). A few large regions without coverage of NCs appear (Figure 31), especially at $7 \mathrm{~V}$. The corresponding current images show a rather different 
appearance from that of $10 \mathrm{SCN}$. At $3 \mathrm{~V}$, the current image is mainly occupied by blue-white and green-yellow regions (Figure 3j). At $5 \mathrm{~V}$, small red domains start to increase and green-yellow spots dominate the image (Figure 3k), suggesting efficient carrier injection into NCs. This is in accord with the optimal device performance near $5 \mathrm{~V}$. At $7 \mathrm{~V}$, most area of the image is blue-white with a few large red spots (Figure 31), suggesting enhanced leakage at high voltage and lower radiative recombination efficiency at high current density. This is in line with the decreased average current from $\sim 33 \mathrm{pA}$ to $\sim 18 \mathrm{pA}$ (Figure 3n), while the maximum current increases from $\sim 0.8 \mathrm{nA}$ to $\sim 1.8 \mathrm{nA}$ when the voltage is increased from $5 \mathrm{~V}$ to $7 \mathrm{~V}$ (Figure 3o). The formation of large uncovered regions together with localized high current density at $7 \mathrm{~V}$ may explain the efficiency roll-off at voltage higher than $6 \mathrm{~V}$ in 30SCN LED. Thus, morphology deterioration of NC film with increasing applied voltage, which may lead to enhanced leakage, could be a reason for efficiency roll-off in LED at high current density [39], as predicted in a report [35] on $\mathrm{CsPbBr}_{3}$ thin film-based LED. Furthermore, stable morphology of $\mathrm{NC}$ film or stable NC distribution in NC film is expected to be able to relieve efficiency roll-off. This is in agreement with some reports $[13,40]$ on embedding perovskite in a matrix, in which the perovskite NCs or pArticles were fixed in the matrix and morphologies of the films are more stable during operation.

Comparing the average current of 10SCN and 30SCN samples at different voltages, it is clear that the initial average currents at $3 \mathrm{~V}$ are almost the same (Figure S6). However, when the current increases to $5 \mathrm{~V}$, the average current in 10SCN significantly rises from $\sim 18 \mathrm{pA}$ to $\sim 84 \mathrm{pA}$ with a large uncertainty range (Figure S6), while the average current in 30SCN increases only from $\sim 19 \mathrm{pA}$ to $\sim 33 \mathrm{pA}$ (Figure 3n). With higher voltage of $7 \mathrm{~V}$, the average current further increases to $\sim 125 \mathrm{pA}$ in 10SCN and decreases to $18 \mathrm{pA}$ in 30SCN. The maximum current follows a similar trend to the average current (Figure 3o). In respect of 10SCN sample, the maximum current reaches $\sim 2.5 \mathrm{nA}$ and $\sim 5 \mathrm{nA}$ at $5 \mathrm{~V}$ and $7 \mathrm{~V}$, respectively. In contrast, the maximum current is merely $\sim 1.8 \mathrm{nA}$ at $7 \mathrm{~V}$ in the $30 \mathrm{SCN}$ sample. Thus, the higher average current together with uncovered regions in 10SCN may be the reason for the lower device performance of 10SCN than that of 30SCN, in which the current increases more smoothly and few uncovered large regions are formed when the applied voltage is less than $7 \mathrm{~V}$.

The stability of the devices was also studied. Figure 4 presents EL under applied voltage of $6 \mathrm{~V}$ from both fresh and aged devices of 10SCN, 30SCN and 50SCN. With PL spectra of fresh films as comparison, it appears that the EL peak of the fresh 30SCN device is the same as corresponding PL peak (Figure 4b). The EL peaks of the fresh 10SCN and 50SCN devices are $1.5 \mathrm{~nm}$ and $0.8 \mathrm{~nm}$ red-shifted with respect to their PL peaks (Figure $4 \mathrm{a}, \mathrm{c}$ ), respectively. When comparing the EL peaks of both fresh and aged devices, all devices display a stable EL peak position or exhibit a red shift (Figure 4d). For instance, for the 10SCN devices, there is a red shift from $\sim 519 \mathrm{~nm}$ to $\sim 523 \mathrm{~nm}$ on aging. On the other hand, the 30SCN devices show only a minor red-shift, if any, from $\sim 518 \mathrm{~nm}$ to $\sim 519$, and the 50SCN devices exhibit no red shift at all, suggesting that more SCN within the considered range is favorable for stability of $\mathrm{CsPbBr}_{3} \mathrm{NCs}$ in the device. In addition, the FWHMs of the EL peaks of 30SCN and 50SCN show negligible change between the fresh and aged devices (Figure 4e). The stable EL spectra of the 30SCN and 50SCN devices at $6 \mathrm{~V}$ are also reflected in the CIE coordinate system (Figure S7), indicating that the treated NCs in the 30SCN and 50SCN devices are rather stable even after storage for four months.

Furthermore, EL peaks and corresponding FWHM at different applied voltages were analyzed for the fresh and aged devices (Figure S8a,b). There are slight blue shifts in the EL peaks and gradual increases in the FWHM with increasing applied voltages in the fresh devices, which could be attributed to a field-induced quantum confinement Stark effect and an enhanced longitudinal optical-phonon coupling at high external field $[5,41]$, respectively. In the case of the aged devices, the FWHM of the EL peaks in 10SCN and 30SCN devices decrease slightly with increasing voltage, while it increases slightly in 50SCN. The EL spectra of the fresh and aged 30SCN devices at different voltages are shown in Figure S8c,d.

The performance of the aged devices were characterized and the statistic results are shown in Figure S9. The aged 30SCN devices still show a higher average EQE than those of 10SCN and 50SCN 
aged devices. In pArticular, the champion EQE of the aged $30 \mathrm{SCN}$ is $1.9 \%$ at $5.6 \mathrm{~V}$, higher than that of the fresh champion device (Figure S4). The higher EQE of the aged device is due to lower current density. According to our research on conductivity of fresh and aged NC films, the aged NC films show a relatively higher conductivity than the fresh devices, which suggests assignment of the decreased current density in the aged devices to degradation of organic transporting layers and corresponding decreased carrier mobility. Statistic results on performance of 41 aged devices based on $30 \mathrm{SCN} \mathrm{CsPbBr} 3$ NCs but with variation in spin coating speed of different layers are shown in Table S2. These results confirm the high stability of the 30SCN devices.
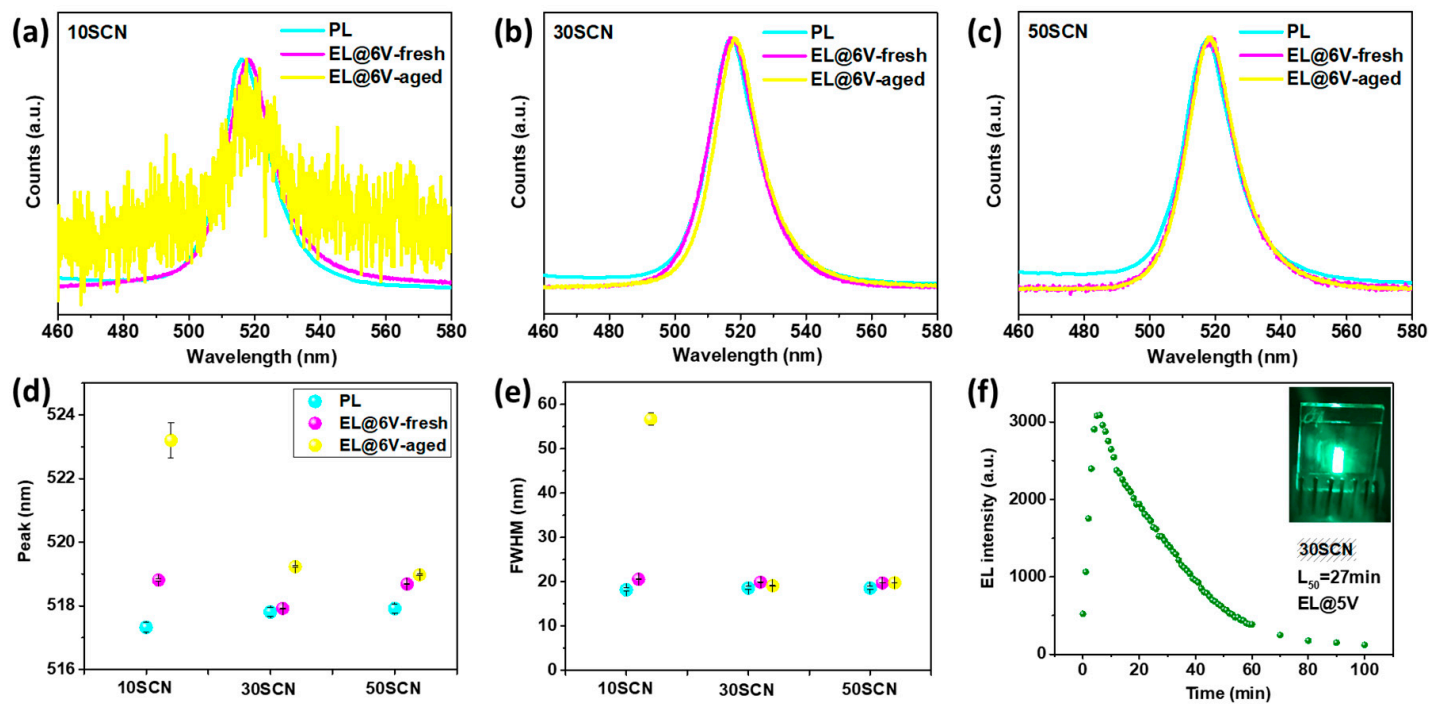

Figure 4. Comparison of PL and EL spectra of (a) 10SCN, (b) $30 \mathrm{SCN}$, and (c) 50SCN NC films and fresh as well as aged devices; Comparison of (d) PL and EL peaks; and (e) FWHM of the PL and EL peaks; (f) Evolution of EL intensity as a function of running time of the 30SCN aged device. The inset is a capture of the device during the operative stability test.

Additionally, operational stability of the $30 \mathrm{SCN}$ aged device was measured at $5 \mathrm{~V}$. There is an initial increase in the EL intensity within the first 6 min (Figure 4f), then the EL intensity shows an exponential deterioration. The half-lifetime (L50) is $\sim 27 \mathrm{~min}$, showing competitive stability in $\mathrm{CsPbBr}_{3}$ NC-based LED (Table S3). EL peaks collected every one minute (Figure S10) are shown to stably fluctuate around $518 \mathrm{~nm}$ throughout the measurement, indicating stable emission property of the $30 \mathrm{SCN}$ aged device. Inset of the Figure $4 \mathrm{f}$ displays the running device during the measurement.

\section{Conclusions}

In summary, green LEDs were fabricated based on $\mathrm{CsPbBr}_{3} \mathrm{NCs}$ synthesized with lead acetate and benzoyl bromide as source of lead and bromine. Softer Lewis acid, ammonium thiocyanate, was used to treat $\mathrm{CsPbBr}_{3}$ NCs before purification with polar solvent. The $30 \mathrm{SCN}$ device showed the best performance, and we investigated possible reasons for the score of this champion device. Morphology and current distribution mapping of NC film under applied voltages were studied by C-AFM. The results show that performance of NC-based LEDs strongly depends on the distribution of NCs in the emissive layer, and that the efficiency roll-off in the NC-based LEDs at high current density can be attributed to displacement and deterioration of NC film with increasing applied voltages. Characterization of fresh and aged devices as well as measurement of running time at $5 \mathrm{~V}$ shows relatively high stability of the $30 \mathrm{SCN}$ device. The current work proposes a facile route to treat sensitive perovskite NCs and to fabricate perovskite NC-based LED with high stability. Moreover, a new evidence was supplied for the efficiency roll-off in NC LED. 
Supplementary Materials: The following are available online at http://www.mdpi.com/1996-1944/13/2/367/s1. Figure S1. TEM images and size distributions of NCs in (a,b) 0SCN, (c,d) 10SCN, (e,f) 30SCN, and (g-k) 50SCN NC solutions. Comparison of (i) fitted average size with FWHM of fitting peaks as error. (j) XRD pAtterns of the NCs; Figure S2. SEM cross-section of (a) 10SCN; (b) 30SCN; and (c) 50SCN devices. From bottom to top, the layers include ITO/PEDOT:PSS + poly-TPD $(\sim 60 \mathrm{~nm}) / \mathrm{CPB}$ NC $(\sim 30 \mathrm{~nm}) / \mathrm{TPBi}(\sim 45 \mathrm{~nm})$; Figure S3. (a) Current density of 30SCN device as a function of driving voltage; (b) EL spectra of 30SCN device at different driving voltages; Figure S4. Comparison of (a) current density as a function of driving voltage; (b) EQE, (c) luminescence of OSCN and 30SCN fresh and aged devices as a function of current density; (d) EL spectra of 0SCN and 30SCN aged devices; Figure S5. (a) Visible light absorption plots; (b) PL; (c) decay lifetime; and (d) fitted decay lifetime and PLQY of NC films on PEDOT:PSS/Poly-TPD substrates; Figure S6. Comparison of average current of 10SCN and 30SCN NC films at different applied voltages; Figure S7. EL positions of fresh and aged devices in a CIE coordinate system; Figure S8. Comparison of (a) EL peaks and (b) FWHM of the EL peaks of fresh and aged devices. EL spectra of 30SCN (c) fresh and (d) aged devices; Figure S9. Statistics of (a) maximum EQE, (b) maximum luminescence, (c) maximum current efficiency, and (d) current density at the maximum EQE of aged devices; Figure S10. (a) EL spectra and (b) evolution of the EL peaks collected during operation stability test of 30SCN aged device; Table S1. Summary of LED performance in literature; Table S2. Statistics of LED performance of the fresh and aged devices; Table S3. Statistics of performance of 41 aged 30SCN-based LEDs.

Author Contributions: Conceptualization, F.C.; methodology, K.M.B. and M.I.; synthesis, F.C.; characterization, F.C., S.L. and M.S.; data analysis, F.C., writing—original draft preparation, F.C.; writing-review and editing, M.S.; critical discussion, F.C. and M.S. All authors have read and agreed to the published version of the manuscript.

Funding: This research received no external funding.

Acknowledgments: Mirko Prato is kindly acknowledged for useful discussion. Quinten A. Akkerman is kindly acknowledged for help with preparation of $\mathrm{NH}_{4} \mathrm{SCN}$ solution. Zhiya Dang is kindly acknowledged for help with HAADF-EDS. Francesco Di Stasio is kindly acknowledged for useful advice in revision of illustrations.

Conflicts of Interest: The authors declare no conflict of interest.

\section{References}

1. Protesescu, L.; Yakunin, S.; Bodnarchuk, M.I.; Krieg, F.; Caputo, R.; Hendon, C.H.; Yang, R.X.; Walsh, A.; Kovalenko, M.V. Nanocrystals of Cesium Lead Halide Perovskites $\left(\mathrm{CsPbX}_{3}, \mathrm{X}=\mathrm{Cl}, \mathrm{Br}\right.$, and I): Novel Optoelectronic Materials Showing Bright Emission with Wide Color Gamut. Nano Lett. 2015, 15, 3692-3696. [CrossRef]

2. Manser, J.S.; Christians, J.A.; Kamat, P.V. Intriguing Optoelectronic Properties of Metal Halide Perovskites. Chem. Rev. 2016, 116, 12956-13008. [CrossRef]

3. Shamsi, J.; Urban, A.S.; Imran, M.; de Trizio, L.; Manna, L. Metal Halide Perovskite Nanocrystals: Synthesis, Post-Synthesis Modifications, and Their Optical Properties. Chem. Rev. 2019, 119, 3296-3348. [CrossRef]

4. Yan, F.; Demir, H.V. LEDs using halide perovskite nanocrystal emitters. Nanoscale 2019, 11, 11402-11412. [CrossRef] [PubMed]

5. Shi, Z.; Li, S.; Li, Y.; Ji, H.; Li, X.; Wu, D.; Xu, T.; Chen, Y.; Tian, Y.; Zhang, Y.; et al. Strategy of Solution-Processed All-Inorganic Heterostructure for Humidity/Temperature-Stable Perovskite Quantum Dot Light-Emitting Diodes. ACS Nano 2018, 12, 1462-1472. [CrossRef] [PubMed]

6. Song, J.; Fang, T.; Li, J.; Xu, L.; Zhang, F.; Han, B.; Shan, Q.; Zeng, H. Organic-Inorganic Hybrid pAssivation Enables Perovskite QLEDs with an EQE of 16.48. Adv. Mater. 2018, 30, e1805409. [CrossRef] [PubMed]

7. Zhang, X.; Lin, H.; Huang, H.; Reckmeier, C.; Zhang, Y.; Choy, W.C.H.; Rogach, A.L. Enhancing the Brightness of Cesium Lead Halide Perovskite Nanocrystal Based Green Light-Emitting Devices through the Interface Engineering with Perfluorinated Ionomer. Nano Lett. 2016, 16, 1415-1420. [CrossRef] [PubMed]

8. Yu, H.; Tian, G.; Xu, W.; Wang, S.; Zhang, H.; Niu, J.; Chen, X. Green Light-Emitting Devices Based on Perovskite $\mathrm{CsPbBr}_{3}$ Quantum Dots. Front. Chem. 2018, 6. [CrossRef]

9. Wang, L.; Liu, B.; Zhao, X.; Demir, H.V.; Gu, H.; Sun, H. Solvent-Assisted Surface Engineering for High-Performance All-Inorganic Perovskite Nanocrystal Light-Emitting Diodes. ACS Appl. Mater. Interfaces 2018, 10, 19828-19835. [CrossRef]

10. Pan, J.; Quan, L.N.; Zhao, Y.; Peng, W.; Murali, B.; Sarmah, S.P.; Yuan, M.; Sinatra, L.; Alyami, N.M.; Liu, J.; et al. Highly Efficient Perovskite-Quantum-Dot Light-Emitting Diodes by Surface Engineering. Adv. Mater. 2016, 28, 8718-8725. [CrossRef]

11. Li, G.; Huang, J.; Li, Y.; Tang, J.; Jiang, Y. Highly bright and low turn-on voltage $\mathrm{CsPbBr}_{3}$ quantum dot LEDs via conjugation molecular ligand exchange. Nano Res. 2018, 12, 109-114. [CrossRef] 
12. Chiba, T.; Hoshi, K.; Pu, Y.-J.; Takeda, Y.; Hayashi, Y.; Ohisa, S.; Kawata, S.; Kido, J. High-Efficiency Perovskite Quantum-Dot Light-Emitting Devices by Effective Washing Process and Interfacial Energy Level Alignment. ACS Appl. Mater. Interfaces 2017, 9, 18054-18060. [CrossRef] [PubMed]

13. Li, G.; Rivarola, F.W.R.; Davis, N.J.L.K.; Bai, S.; Jellicoe, T.C.; de la Peña, F.; Hou, S.; Ducati, C.; Gao, F.; Friend, R.H.; et al. Highly Efficient Perovskite Nanocrystal Light-Emitting Diodes Enabled by a Universal Crosslinking Method. Adv. Mater. 2016, 28, 3528-3534. [CrossRef]

14. Shi, Z.; Li, Y.; Li, S.; Li, X.; Wu, D.; Xu, T.; Tian, Y.; Chen, Y.; Zhang, Y.; Zhang, B.; et al. Localized Surface Plasmon Enhanced All-Inorganic Perovskite Quantum Dot Light-Emitting Diodes Based on Coaxial Core/Shell Heterojunction Architecture. Adv. Funct. Mater. 2018, 28, 1707031. [CrossRef]

15. Song, J.; Li, J.; Li, X.; Xu, L.; Dong, Y.; Zeng, H. Quantum Dot Light-Emitting Diodes Based on Inorganic Perovskite Cesium Lead Halides $\left(\mathrm{CsPbX}_{3}\right)$. Adv. Mater. 2015, 27, 7162-7167. [CrossRef]

16. Zhang, X.; Xu, B.; Zhang, J.; Gao, Y.; Zheng, Y.; Wang, K.; Sun, X.W. All-Inorganic Perovskite Nanocrystals for High-Efficiency Light Emitting Diodes: Dual-Phase $\mathrm{CsPbBr}_{3}-\mathrm{CsPb}_{2} \mathrm{Br}_{5}$ Composites. Adv. Funct. Mater. 2016, 26, 4595-4600. [CrossRef]

17. Li, J.; Xu, L.; Wang, T.; Song, J.; Chen, J.; Xue, J.; Dong, Y.; Cai, B.; Shan, Q.; Han, B.; et al. 50-Fold EQE Improvement up to $6.27 \%$ of Solution-Processed All-Inorganic Perovskite CsPbBr3 QLEDs via Surface Ligand Density Control. Adv. Mater. 2017, 29, 1603885. [CrossRef] [PubMed]

18. Chen, W.; Tang, X.; Wangyang, P.; Yao, Z.; Zhou, D.; Chen, F.; Li, S.; Lin, H.; Zeng, F.; Wu, D.; et al. Surface-Passivated Cesium Lead Halide Perovskite Quantum Dots: Toward Efficient Light-Emitting Diodes with an Inverted Sandwich Structure. Adv. Opt. Mater. 2018, 6, 1800007. [CrossRef]

19. Tan, Y.; Zou, Y.; Wu, L.; Huang, Q.; Yang, D.; Chen, M.; Ban, M.; Wu, C.; Wu, T.; Bai, S.; et al. Highly Luminescent and Stable Perovskite Nanocrystals with Octylphosphonic Acid as a Ligand for Efficient Light-Emitting Diodes. ACS Appl. Mater. Interfaces 2018, 10, 3784-3792. [CrossRef]

20. Emre, Y.; Zhenyu, Y.; Oleksandr, V.; Younghoon, K.; Grant, W.; Andres, C.J.; Pongsakorn, K.; Mingjian, Y.; Xiwen, G.; Fengiia, F.; et al. Amine-Free Synthesis of Cesium Lead Halide Perovskite Quantum Dots for Efficient Light-Emitting Diodes. Adv. Funct. Mater. 2016, 26, 8757-8763.

21. Liu, P.; Chen, W.; Wang, W.; Xu, B.; Wu, D.; Hao, J.; Cao, W.; Fang, F.; Li, Y.; Zeng, Y.; et al. Halide-Rich Synthesized Cesium Lead Bromide Perovskite Nanocrystals for Light-Emitting Diodes with Improved Performance. Chem. Mater. 2017, 29, 5168-5173. [CrossRef]

22. Krieg, F.; Ochsenbein, S.T.; Yakunin, S.; Brinck, S.T.; Aellen, P.; Süess, A.; Clerc, B.; Guggisberg, D.; Nazarenko, O.; Shynkarenko, Y.; et al. Colloidal $\mathrm{CsPbX}_{3}(\mathrm{X}=\mathrm{Cl}, \mathrm{Br}$, I) Nanocrystals 2.0: Zwitterionic Capping Ligands for Improved Durability and Stability. ACS Energy Lett. 2018, 3, 641-646. [CrossRef] [PubMed]

23. Imran, M.; Caligiuri, V.; Wang, M.; Goldoni, L.; Prato, M.; Krahne, R.; de Trizio, L.; Manna, L.J. Benzoyl Halides as Alternative Precursors for the Colloidal Synthesis of Lead-Based Halide Perovskite Nanocrystals. Am. Chem. Soc. 2018, 140, 2656-2664. [CrossRef] [PubMed]

24. Lin, K.; Xing, J.; Quan, L.N.; de Arquer, F.P.G.; Gong, X.; Lu, J.; Xie, L.; Zhao, W.; Zhang, D.; Yan, C.; et al. Perovskite light-emitting diodes with external quantum efficiency exceeding 20 per cent. Nature 2018, 562, 245-248. [CrossRef]

25. Tong, J.; Song, Z.; Kim, D.H.; Chen, X.; Chen, C.; pAlmstrom, A.F.; Ndione, P.F.; Reese, M.O.; Dunfield, S.P.; Reid, O.G.; et al. Carrier lifetimes of $>1 \mu \mathrm{s}$ in $\mathrm{Sn}-\mathrm{Pb}$ perovskites enable efficient all-perovskite tandem solar cells. Science 2019, 364, 475-479. [CrossRef]

26. Jiang, Q.; Rebollar, D.; Gong, J.; Piacentino, E.L.; Zheng, C.; Xu, T. Pseudohalide-Induced Moisture Tolerance in Perovskite $\mathrm{CH}_{3} \mathrm{NH}_{3} \mathrm{~Pb}(\mathrm{SCN})_{2}$ I Thin Films. Angew. Chem. Int. Ed. 2015, 54, 7617-7620. [CrossRef]

27. Zhang, R.; Li, M.; Huan, Y.; Xi, J.; Zhang, S.; Cheng, X.; Wu, H.; Peng, W.; Bai, Z.; Yan, X. A potassium thiocyanate additive for hysteresis elimination in highly efficient perovskite solar cells. Inorg. Chem. Front. 2019, 6, 434-442. [CrossRef]

28. Lou, Y.; Niu, Y.; Yang, D.; Xu, Q.; Hu, Y.; Shen, Y.; Ming, J.; Chen, J.; Zhang, L.; Zhao, Y. Rod-shaped thiocyanate-induced abnormal band gap broadening in $\mathrm{SCN}-$ doped $\mathrm{CsPbBr}_{3}$ perovskite nanocrystals. Nano Res. 2018, 11, 2715-2723. [CrossRef]

29. Koscher, B.A.; Swabeck, J.K.; Bronstein, N.D.; Alivisatos, A.P. Essentially Trap-Free CsPbBr3 Colloidal Nanocrystals by Postsynthetic Thiocyanate Surface Treatment. J. Am. Chem. Soc. 2017, 139, 6566-6569. [CrossRef] 
30. Nenon, D.P.; Pressler, K.; Kang, J.; Koscher, B.A.; Olshansky, J.H.; Osowiecki, W.T.; Koc, M.A.; Wang, L.-W.; Alivisatos, A.P. Design Principles for Trap-Free $\mathrm{CsPbX}_{3}$ Nanocrystals: Enumerating and Eliminating Surface Halide Vacancies with Softer Lewis Bases. J. Am. Chem. Soc. 2018, 140, 17760-17772. [CrossRef]

31. Lu, M.; Guo, J.; Lu, P.; Zhang, L.; Zhang, Y.; Dai, Q.; Hu, Y.; Colvin, V.L.; Yu, W.W. Ammonium Thiocyanate-Passivated CsPbI3 Perovskite Nanocrystals for Efficient Red Light-Emitting Diodes. J. Phys. Chem. C 2019, 123, 22787-22792. [CrossRef]

32. Zou, W.; Li, R.; Zhang, S.; Liu, Y.; Wang, N.; Cao, Y.; Miao, Y.; Xu, M.; Guo, Q.; Di, D.; et al. Minimising efficiency roll-off in high-brightness perovskite light-emitting diodes. Nat. Commun. 2018, 9, 608. [CrossRef] [PubMed]

33. Bae, W.K.; pArk, Y.-S.; Lim, J.; Lee, D.; pAdilha, L.A.; McDaniel, H.; Robel, I.; Lee, C.; Pietryga, J.M.; Klimov, V.I. Controlling the influence of Auger recombination on the performance of quantum-dot light-emitting diodes. Nat. Commun. 2013, 4, 2661. [CrossRef] [PubMed]

34. Perumal, A.; Shendre, S.; Li, M.; Tay, Y.K.E.; Sharma, V.K.; Chen, S.; Wei, Z.; Liu, Q.; Gao, Y.; Buenconsejo, P.J.S.; et al. High brightness formamidinium lead bromide perovskite nanocrystal light emitting devices. Sci. Rep. 2016, 6, 36733. [CrossRef] [PubMed]

35. Wang, H.; Zhang, X.; Wu, Q.; Cao, F.; Yang, D.; Shang, Y.; Ning, Z.; Zhang, W.; Zheng, W.; Yan, Y.; et al. Trifluoroacetate induced small-grained $\mathrm{CsPbBr}_{3}$ perovskite films result in efficient and stable light-emitting devices. Nat. Commun. 2019, 10, 665. [CrossRef]

36. Imran, M.; Ijaz, P.; Goldoni, L.; Maggioni, D.; Petralanda, U.; Prato, M.; Almeida, G.; Infante, I.; Manna, L. Simultaneous cationic and anionic ligand exchange for colloidally stable $\mathrm{CsPbBr}_{3}$ nanocrystals. ACS Energy Lett. 2019, 4, 819-824. [CrossRef]

37. Gao, F.; Li, C.; Qin, L.; Zhu, L.; Huang, X.; Liu, H.; Liang, L.; Hou, Y.; Lou, Z.; Hu, Y.; et al. Enhanced performance of tin halide perovskite solar cell by addition of lead thiocyanate. RSC Adv. 2018, 8, 14025-14030. [CrossRef]

38. Shi, Y.; Wu, W.; Dong, H.; Li, G.; Xi, K.; Divitini, G.; Ran, C.; Yuan, F.; Zhang, M.; Jiao, B.; et al. A Strategy for Architecture Design of Crystalline Perovskite Light-Emitting Diodes with High Performance. Adv. Mater. 2018, 30, 1800251. [CrossRef]

39. Sun, G.; Lu, K.; Kun, F. Percolation-induced conductor-insulator transition in a system of metal spheres in a dielectric fluid. Phys. Rev. E 2011, 83, 041405. [CrossRef]

40. Giuri, A.; Yuan, Z.; Miao, Y.; Wang, J.; Gao, F.; Sestu, N.; Saba, M.; Bongiovanni, G.; Colella, S.; Corcione, C.E.; et al. Ultra-Bright Near-Infrared Perovskite Light-Emitting Diodes with Reduced Efficiency Roll-off. Sci. Rep. 2018, 8, 15496. [CrossRef]

41. Wood, V.; pAnzer, M.J.; Caruge, J.-M.; Halpert, J.E.; Bawendi, M.G.; Bulović, V. Air-Stable Operation of Transparent, Colloidal Quantum Dot Based LEDs with a Unipolar Device Architecture. Nano Lett. 2010, 10, 24-29. [CrossRef] [PubMed]

(C) 2020 by the authors. Licensee MDPI, Basel, Switzerland. This article is an open access article distributed under the terms and conditions of the Creative Commons Attribution (CC BY) license (http://creativecommons.org/licenses/by/4.0/). 